\title{
Percutaneous Intrapericardial Interventions Using a Highly Articulated Robotic Probe
}

\author{
Amir Degani ${ }^{\mathrm{a}}$ and Howie Choset ${ }^{\mathrm{a}, \mathrm{c}}$ \\ ${ }^{a}$ The Robotics Institute \\ Carnegie Mellon University \\ Pittsburgh PA 15213-3891, USA \\ degani@cmu.edu,choset@cs.cmu.edu
}

\author{
Alon Wolf ${ }^{\mathrm{b}, \mathrm{a}, \mathrm{c}}$ \\ ${ }^{b}$ Dept. of Mechanical Engineering \\ Technion Israel Institute of Technology \\ Technion City, Haifa 32000, Israel \\ alonw@tx.technion.ac.il
}

\author{
Takeyoshi Ota ${ }^{\mathrm{c}}$ and Marco A. Zenati ${ }^{\mathrm{c}, \mathrm{a}}$ \\ ${ }^{c}$ Division of Cardiothoracic Surgery \\ University of Pittsburgh Medical Center \\ Pittsburgh ,PA 15213-2582, USA \\ otat@upmc.edu,zenatim@upmc.edu
}

\begin{abstract}
In order to overcome the limitations of currently available assistive technologies for minimally invasive surgery (MIS), we have developed a novel highly articulated robotic probe (HARP) that can exploit its snake-like structure to navigate in a confined anatomical environment while minimally interacting with the environment along its path. We believe that for procedures involving epicardial interventions on the beating heart, cardiac MIS can be effectively realized with the HARP, entering the pericardial cavity through a subxiphoid port, reaching remote intrapericardial locations on the epicardium without causing hemodynamic and electrophysiologic interference and delivering therapeutic interventions under the direct control of the surgeon.
\end{abstract}

Index Terms - Cardiac Surgery, Medical Robot, Minimally Invasive Surgery.

\section{INTRODUCTION}

The clinical benefits to patients are profound when an "open" procedure can be made minimally invasive. By definition, performing any procedure less invasively results in less soft tissue disruption, with the effects of reduced pain, faster healing and recovery, and fewer complications. Documented advantages of less invasive procedures include smaller incisions and fewer injuries to major blood vessels and nerves [1]; other benefits reported include reduced blood loss and decreased post-operative pain [2]; shorter hospital stays and faster return to normal activity for the patient [3]. Further, despite the higher capital expense of equipment needed to operate in a minimally invasive fashion, overall costs of minimally invasive procedures can actually be significantly lower $[4$, 5].

In order to overcome the limitations of currently available assistive technologies for minimally invasive surgery (MIS), we developed and tested a proof of concept $12 \mathrm{~mm}$ in diameter, $300 \mathrm{~mm}$ long, three-dimensional highly articulated robotic probe (HARP) for MIS (Fig. 1). We believe that the HARP can be applied on a broad range of medical procedures. We use cardiac MIS as a focal application for this mechanism. Cardiac surgery is different from other surgical procedures because the large sternotomy incision required to access the heart requires general endotracheal anesthesia. The heart-lung machine that is required for open-heart surgery (e.g. valve repair) adds further morbidity. If for that reason only, performing an epicardial intervention in a less invasive manner will dramatically improve recovery and decrease risks involved with the current procedures. Some of the potential intrapericardial therapies that the HARP can deliver include: cell transplantation by intramyocardial injection, epicardial ablation, and epicardial lead placement for resynchronization.

The HARP mechanism, described in this paper, differs from previous devices in its ability to form a curve in three-dimensions with only six actuators. We present an overview of existing medical devices, show the mechanical design of the HARP and report our preliminary experiments on live pigs.

\section{PREVIOUS Work}

\section{A. Minimally Invasive Cardiac Surgery}

As mentioned in the introduction, we are using cardiac MIS as our focal application; we chose cardiac surgery partially because of the expertise of our team. The subxiphoid route, depicted in Fig. 2, is an ideal point of entry for cardiac procedures because its use avoids the need for a large sternotomy incision. Zenati et al. are among the first to demonstrate this approach for epicardial left heart pacing lead implantation for resynchronization $[6,7]$.

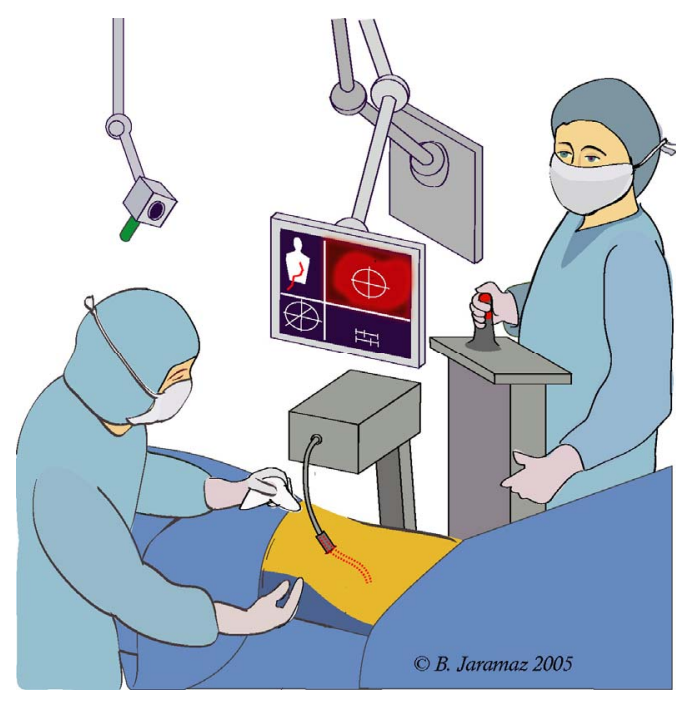

Fig. 1: HARP concept 


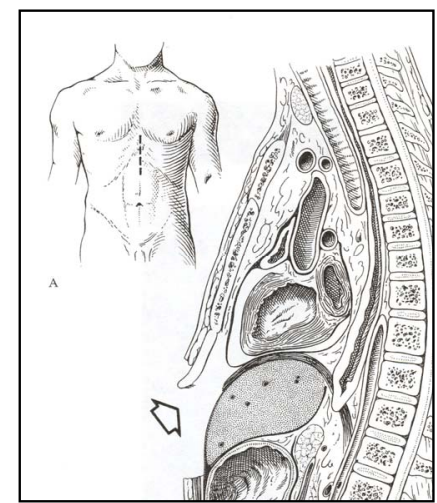

Fig. 2: The subxiphoid route

The subxiphoid videopericardioscopy (SVP) device (Guidant Corporation, Santa Clara, CA) is the only dedicated technology available for endoscopic video exploration of the pericardial cavity. One major problem associated with the present configuration of the SVP device is its rigidity and the significant potential that the compression of the beating heart will trigger a lifethreatening arrhythmia.

Most of the anatomical targets for videopericardioscopy are located in remote areas of the pericardium, away from the entry point in the pericardium below the xiphoid. One such target is the left atrial appendage (LAA), the ligation of which was shown to reduce stroke in atrial fibrillation $[8,9]$. Our $12 \mathrm{~mm}$ in diameter HARP can get to those hard to reach anatomical targets by using its high redundancy and maneuverability to navigate in the confined anatomical environment while minimally interacting with the environment along its path.

\section{B. $\quad$ Related Work in Medical Devices}

Recently there have been successful developments in the medical robotic domain, most notably is the da Vinci system (Intuitive Inc., Sunnyvale, CA) which uses conventional industrial robot arms that grab custom design laparoscopic tools with which MIS can be performed. In this section we will only present a review on laparoscopic technologies and mechanisms relevant to the one described in this paper.

Endoscopic instruments are the main tools used today in minimally invasive surgery (MIS). A typical endoscope consists of a long $10 \mathrm{~mm}$ in diameter tube with a length of 70 to $180 \mathrm{~mm}$. Although these devices have a steerable tip which allows them to be directed inside the body, current endoscopic tools cannot maneuver in very small and geometrically complex spaces. To overcome this problem numerous works have been presented trying to increase the maneuverability of active catheters and endoscopes. Much of these works have focused on developing a new form of actuation, such as shape memory allows (SMA) $[10,11]$ and electric polymer artificial muscles (EPAM) $[12,13]$.

The Laboratoire de Robotique de Paris (LRP) developed an $8 \mathrm{~mm}$ in diameter snake-like mechanism which is formed by a sequence of segments articulated by SMA actuated pin joints [14]. Hirose [15] also used an SMA spring and wire actuation to make a small surgical device; the novelty of his mechanism is that it overcomes hysteresis commonly found in SMA's.

In [16] the researchers developed a $2.8 \mathrm{~mm}$ in diameter active catheter based on silicon micromachining. This multilane manipulator is connected by joints made of SMA, fixed at equilateral triangular locations to allow bending in several directions. Other endoscopic tools, based on SMA and piezoelectric technology, are presented in [17-21].

Although SMA's offer a compact alternative to conventional actuators, virtually all SMA tools have relatively low stiffness, and require high activation voltage. This high activation voltage makes heat removal quite challenging.

A different activation concept is presented in [22] and [23]. In these works the authors presented snake-like tools using super-elastic NiTi which has higher stiffness than other SMAs but the heating problem and complexity of multiple degrees of freedom (DOF) is still relevant, resulting in limited DOF mechanisms.

Lately there has been an increase interest in electrostrictive polymer artificial muscle (EPAM) for medical applications [24]. An EPAM based snake-like endoscopic robot was developed at Stanford Research Institute (SRI). The device is composed of several spherical joints attached serially around a concentric spine [12]. Researchers at Pennsylvania State University [13] have also developed a snake-like manipulator using EPAM. Their special design of the actuator allows control of the curvature.

Another popular actuation scheme is wire actuation, such as the arthroscope tool developed at the Santa Anna Laboratory in Pisa, Italy, [25]. The 25-mm long distal section of the arthroscope has a 1-DOF with an angle range of $0^{\circ}$ to $110^{\circ}$.

Perhaps the biggest drawback of wire actuation, SMA, and EPAM actuators is that they do not have the strength to "hold" the device in three dimensions. This means that they cannot form a true three-dimensional curve. Most of these devices were originally designed to operate only inside a luminal or tube-like environment.

\section{HARP DESIGN AND OPERATION}

A prototype of the highly articulated robot for minimally invasive surgery has been built and tested in our lab. The current prototype is $12 \mathrm{~mm}$ in diameter and $300 \mathrm{~mm}$ in length, and can achieve a $7.5 \mathrm{~cm}$ radius of curvature. The choice of $12 \mathrm{~mm}$ is based on available ports. With the feeder mechanism, described below, the overall dimensions of the mechanism are approximately $500 \mathrm{~mm}$ length, $170 \mathrm{~mm}$ width, and $100 \mathrm{~mm}$ height (Fig. 3).

Four cables actuate the probe. The source of actuation is off-board which means that no electric power, heat dissipation, etc. occurs inside the HARP, and hence inside the body, when the HARP is inserted into a patient.

Visual feedback from the distal link is relayed to an external video camera by a $2 \mathrm{~mm}$ diameter fiber optic endoscope attached to the outer mechanism, and displayed on a monitor. 


\section{A. Design Concept}

The HARP design consists of two concentric tubes. We refer to these tubes as inner and outer mechanisms. Each tube can alternate between being rigid or limp. In rigid mode, the mechanism is just that - rigid. In limp mode, the mechanism is highly flexible and thus either assumes the shape of its surroundings or can be reshaped in some other way. The HARP follows an arbitrary curve in threedimensional space by alternating the rigidity/limpness of the outer and inner mechanisms. For example, starting with both inner and outer mechanisms as rigid, the outer mechanism is made limp and advances forward one link. Even though the outer mechanism is limp, it maintains most of its shape because the inner mechanism is rigid. However, since the outer mechanism is indeed limp, it can "steer" its head, the link which was advanced forward. Now, the outer mechanism is made rigid, the inner mechanism is made limp, the inner mechanism advances until it "catches up" to the outer mechanism. The inner mechanism is then made rigid, and the procedure repeats.

Both inner and outer mechanisms consist of rigid cylindrical links connected by a type of spherical joint which can rotate $\pm 15^{\circ}$ range in both degrees of freedom. The links are strung together by cables, three for the outer mechanism and one for the inner mechanism (Fig. 4). The three outer cables of the outer mechanism are $120^{\circ}$ apart, making it possible to steer in any direction, as well as selecting between rigid and limp modes. When the cables are pulled towards the back of the mechanism, the links are pulled towards each other increasing friction between the links eventually causing the outer mechanism to become rigid; when they are relaxed, the outer mechanism becomes limp. A patent on this concept has been submitted.
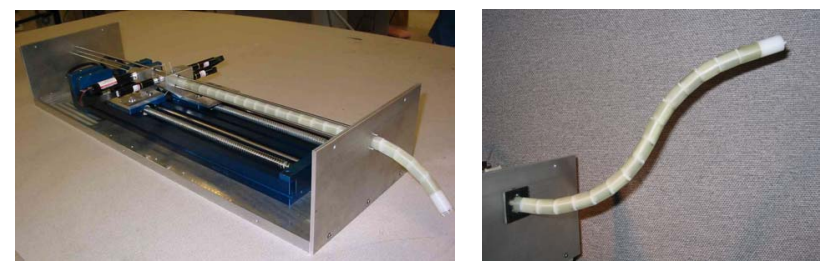

Fig. 3: HARP prototype

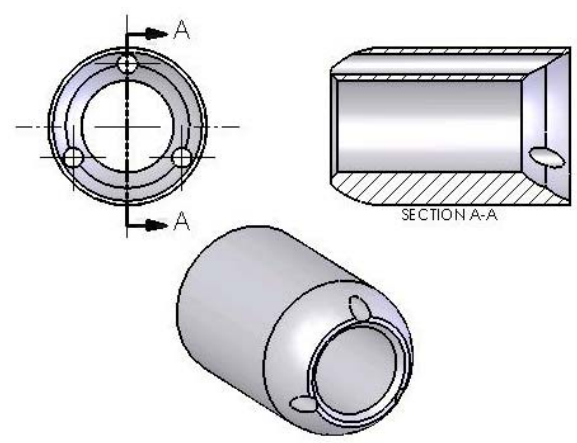

Fig. 4: Single link of the outer mechanism

\section{B. Feeder Design}

The feeding mechanism inserts the HARP into a region of interest. Referring to Fig. 5, the feeder has two movable carriers. A first carrier (c) drives the outer mechanism and a second carrier (e) drives the inner mechanism. Each carrier, and hence the inner and outer mechanisms, are driven independently by separate linear motion systems $\left(a_{1}\right.$, $\mathrm{a}_{2}$ ).

Each of the carriers (c and e) transports motors necessary for controlling the cables of the inner mechanism and outer mechanism. As seen in Fig. 5, the outer mechanism carrier (c) transports three motors (d) which control the tension on the cables of the outer mechanism. The second carrier (e) transports a motor (f) for controlling the tension on the cable of the inner mechanism. HARP's mechanical part list is shown in table I.

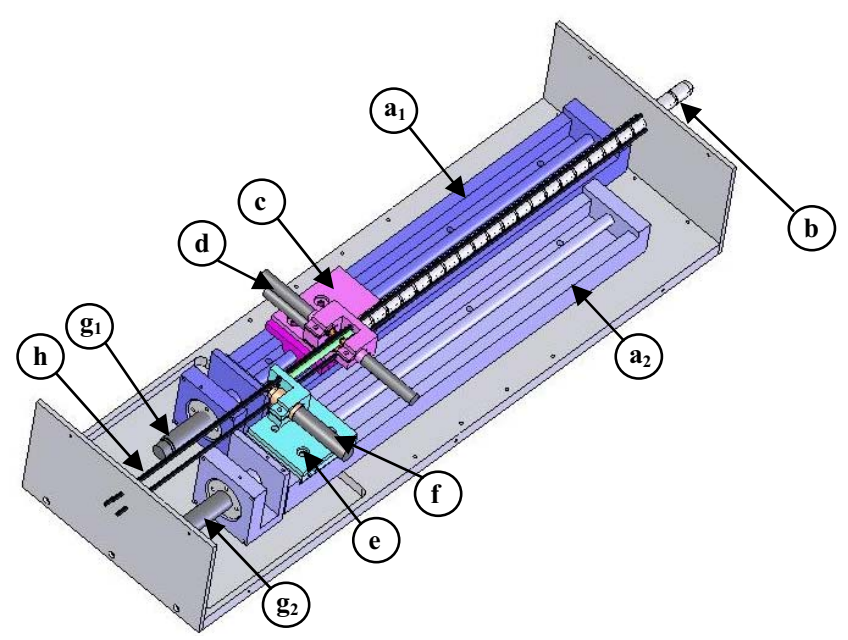

Fig. 5: HARP mechanism assembly

TABLE I

HARP MECHANICAL PART LIST

\begin{tabular}{|l|l|}
\hline a & Linear motion system (x2) \\
\hline b & Inner and outer mechanism \\
\hline c & Outer mechanism carrier \\
\hline d & Actuators for controlling outer mechanism (x3) \\
\hline e & Inner mechanism carrier \\
\hline f & Actuator for controlling the inner mechanism \\
\hline g & Actuator for driving linear system (x2) \\
\hline h & Rods acting as a linear rail for mechanism $(\mathrm{x} 3)$ \\
\hline
\end{tabular}




\section{Applied Loads}

To determine the torques required from the motors, as well as the material of the HARP itself, an extreme or worst-case configuration of the HARP was considered, i.e. a configuration where the actuators have to exert the most torque. Such an extreme configuration occurs when the device is stretched out in a cantilevered position, the outer mechanism is limp, and the inner mechanism supports its own weight as well as the weight of the outer mechanism (Fig. 6a). To estimate the axial force needed to be applied by the cable of the inner mechanism to support this configuration, a simplified model of this extreme configuration was generated. For this simplified model we approximated the system parameters: the outer mechanism link weight as 1.5 grams, the inner mechanism link weight as 0.5 grams, the number of links in each mechanism as 17, the total weight as 34 grams, the total length of the HARP as $300 \mathrm{~mm}$, the outer mechanism outer diameter as $12 \mathrm{~mm}$, and the inner mechanism outer diameter as $6 \mathrm{~mm}$. The weight of the HARP is simplified to a point mass at the center of gravity of the HARP.

The largest torque is exerted on the area between the two proximal links of the mechanism. Therefore we simplified the model to include only one long link that is in contact with the proximal link (Fig. 6b). The cable of the inner mechanism is applied with an axial force, $F$, at the center of the mechanism (Fig. 6b). A free body diagram of the simplified model is shown in Fig. 6c.

The approximated relation between the force $F$ and the torque $\tau$ applied on a spherical surface with radius $r$ and friction coefficient $\mu$ is given in (1).

$\tau=\mu F r \Rightarrow F=\frac{\tau}{\mu \cdot r}=\frac{50 \mathrm{~N} \cdot \mathrm{mm}}{\mu \cdot 3 \mathrm{~mm}} \approx \frac{17 \mathrm{~N}}{\mu}$

It is clear from (1) that the friction coefficient is crucial. When the friction between links is low, the pulling force needed to withstand the mechanism's own weight is enormous. Therefore the material selection of the links is a crucial factor.

In order to estimate the torque required from the motor to pull the cable with the required force $F$, we use the relationship between the motor torque $\left(\tau_{\text {motor }}\right)$, the radius of the pulley $\left(r_{p}\right)$, and the pulling force on the cable $(F)$ :

$\tau_{\text {motor }}=F r_{p} \Leftrightarrow F=\frac{\tau_{\text {motor }}}{r_{p}}$

As observed in (2), in order to output a higher force per given motor torque, a small radius of the pulley is required. We used a machined 10-24 screw as the pulley, which has approximately a $2 \mathrm{~mm}$ radius (Fig. 7 ). In the current design of the HARP the outer mechanism, when in a stiff mode, is able to withstand loads of about one to five Newton (depending on the direction of the load and the configuration of the mechanism).

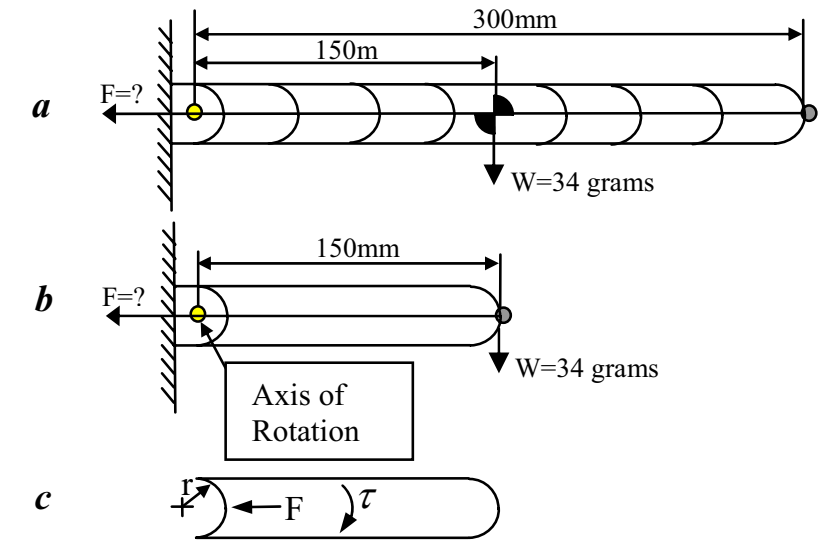

Fig. 6: Schematic of extreme cantilever configuration a. Extreme configuration b. Simplified model c. Free body diagram of extreme configuration

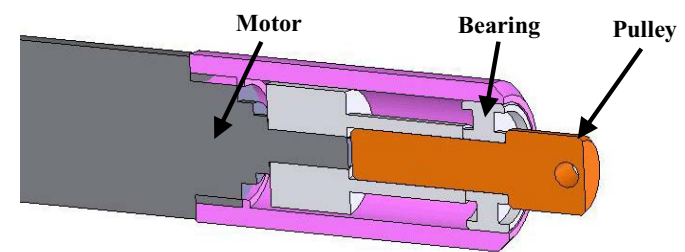

Fig. 7: Cross-section of motor and pulley system

\section{PRELIMINARY EXPERIMENTS}

Using available CAD software and a finite element heart model [26], we have simulated different paths of the probe and described how the HARP navigates and delivers therapies within the intrapericardial space. We have simulated three different paths: 1 . Entering the subxiphoid port, posterior approach to the left atrial appendage (LAA) (Fig. 8a). 2. Entering the subxiphoid port, anterior approach to the LAA (Fig. 8b). 3. Entering the subxiphoid port toward the transverse sinus and exiting through the superior vena cava (Fig. 8c). These simulations were made in order to identify the length and minimal curvatures necessary for the robot to achieve. These simulations were further validated with live pig operations.
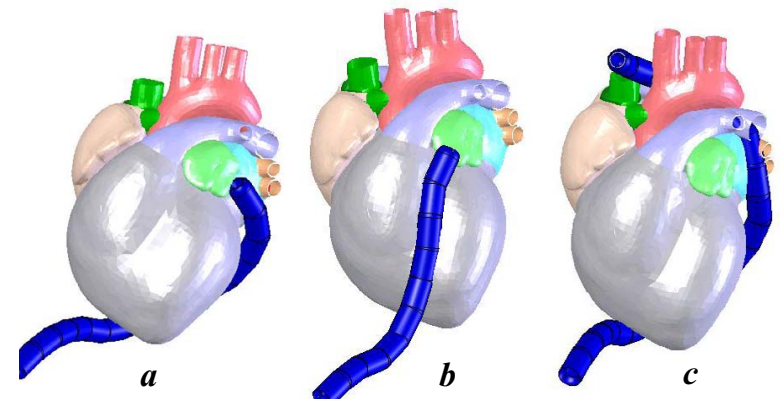

Fig. 8: Simulation of robotic probe on heart model 
The HARP prototype was tested in four different healthy and large $(35-45 \mathrm{~kg})$ pigs. The experiment protocol was approved by the Institutional Animal Care and Use Committee (IACUC) of the University of Pittsburgh. We performed preclinical feasibility testing of the HARP prototype in the open-chest intrapericardial environment and in abdominal laparoscopy.

The thoracic experiments were performed in the anesthetized animals following a median sternotomy. A small $15 \mathrm{~mm}$ opening was created on the pericardium at the junction with the diaphragm; through this opening the HARP was remotely guided to slide between the pericardium and the anterior wall of the right ventricle of the beating heart. The HARP was advanced to the root of the aorta and was then withdrawn out of the pericardium. This task was repeated several times to test deployment speed and amount of resistance by the motion of the beating heart and from the intact pericardium (Fig. 9). Although deployment speeds were not measured precisely, it was concluded that the robot's speed must be slightly increased.

After opening of the pericardium, the HARP was guided through the transverse sinus of the pericardium. This path was successfully completed both with the HARP entering through a subxiphoid access (Fig. 9), and from a right thoracic port (Fig. 10). An endoscopic biopsy forceps was advanced through the HARP's working port to simulate a pericardial biopsy; several specimens of pericardium were successfully harvested through the HARP (Fig. 11a). Furthermore, we were able to insert through the HARP's working port a customized Endoloop ${ }^{\mathrm{TM}}$ (Ethicon Endosurgery), and simulated an LAA ligation (Fig. 11b).

After the completion of the experiment, absence of any gross epicardial damage to the heart was confirmed by visual inspection. No adverse hemodynamic or electrocardiographic interference induced by the motion of the HARP was detected.
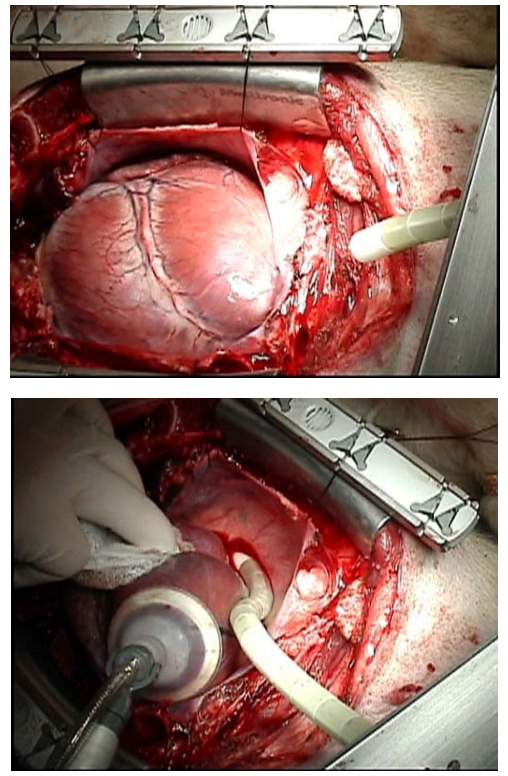

Fig. 9: HARP inserted from the subxiphoid port towards the transverse sinus of the pericardium (the Urchin heart positioner was only used to displace the heart in order to show the hidden mechanism.)
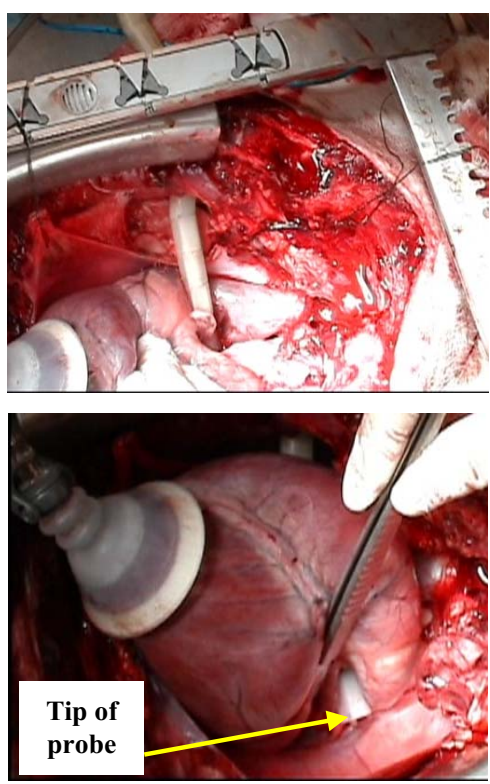

Fig. 10: HARP inserted from the right thoracic port through the transverse sinus of the pericardium.

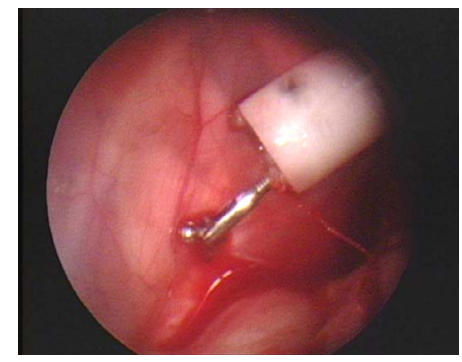

$a$

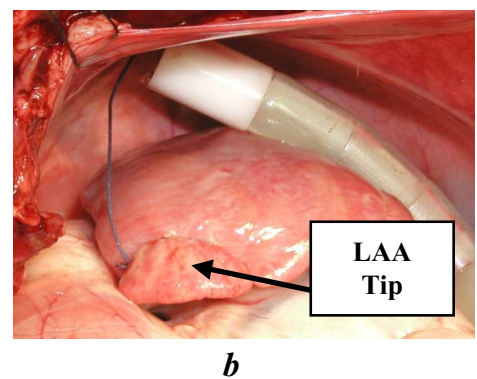

Fig. 11: Inserting tools through the HARP's working port: a. Using biopsy forceps to perform pericardium biopsies.

b. Using Endoloop ${ }^{\mathrm{TM}}$ to ligate the left atrial appendage (LAA).

We also performed an abdominal laparoscopic experiment using a conventional $15 \mathrm{~mm}$ endoscopic port positioned in the right lower abdominal quadrant (Ethicon Endosurgery, Somerville, NJ). Visualization of the HARP was provided by a laparoscope positioned through an additional abdominal port in the left lower quadrant. Insufflation of the abdominal cavity with $\mathrm{CO} 2$ was performed. The HARP was successfully advanced using remote tele-manipulation through the port and into the abdominal cavity (Fig. 12). The HARP was advanced towards the right kidney region after retraction of the small bowel. This experiment was repeated several times using different trajectories. No damage to the intraabdominal structures was realized. 

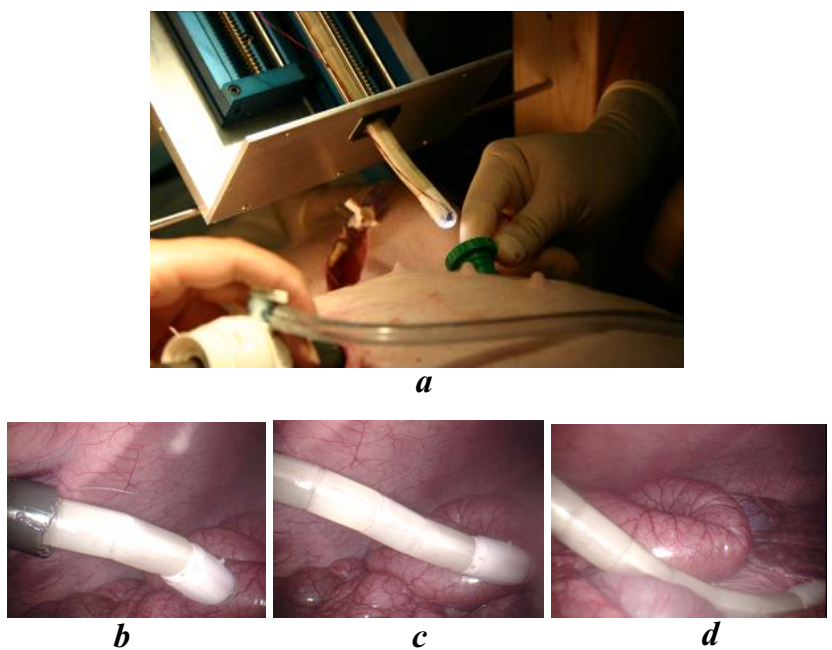

Fig. 12: Harp inserted to the abdominal cavity a. HARP entering the port b-d. HARP inside the abdominal cavity

\section{CONCLUSION AND DISCUSSION}

In this paper we have discussed the motivations for the development of a new system for minimally invasive surgery, and presented the design and preliminary tests of the HARP prototype. We believe the HARP overcomes the limitations of today's MIS tools.

The main advantage of the HARP device, besides its relative small dimensions, is its capability to preserve its previous configuration, i.e. create a curve in threedimension space (Fig. 3). Since, there are no mechanical moving parts within the mechanism itself, it can be made out of virtually any material, including plastic.

Preliminary experiments with our robotic prototype strongly indicate that the design concept is sound. Therefore, we will continue development of the HARP focusing on a smaller diameter, increased stiffness and better curvature capabilities. Furthermore, advanced histological investigation of the epicardial surface and more precise quantitative experiments would also be conducted.

\section{REFERENCES}

[1] T. J. Huang, R. W. Hsu, S. H. Chen, and Y. Y. Lee, "Minimal access surgery in managing anterior lumbar disorders," Clin Orthop Relat Res, pp. 140-7, 2001.

[2] S. M. Nymberg and A. H. Crawford, "Video-assisted thoracoscopic releases of scoliotic anterior spines," AORN journal, vol. 63, pp. 5612, 565-9; 571-5; quiz 576, pass, 1996.

[3] A. Toniato, P. Bernante, G. P. Rosse, A. Piotto, and M. R. Pelizzo, "Laparoscopic versus open adrenalectomy: outcome in 35 consecutive patients," International journal of surgical investigation, vol. 1, pp. 503-7, 2000.

[4] L. H. Cohn, et al., "Minimally invasive cardiac valve surgery improves patient satisfaction while reducing costs of cardiac valve replacement and repair," Annals of surgery, vol. 226, pp. 421-6; discussion 427-8, 1997.
[5] M. L. Montgomery and J. P. Sullivan, "Advances in interventional radiology. The search for less invasive management sparks new approaches," Postgraduate medicine, vol. 109, pp. 93-4, 97-9, 103-4, 2001.

[6] M. A. Zenati, G. Bonanomi, A. K. Chin, and D. Schwartzman, "Left heart pacing lead implantation using subxiphoid videopericardioscopy," Journal of cardiovascular electrophysiology, vol. 14, pp. 949-53, 2003.

[7] C. Leclercq and D. A. Kass, "Retiming the failing heart: principles and current clinical status of cardiac resynchronization," Journal of the American College of Cardiology, vol. 39, pp. 194-201, 2002.

[8] M. A. Zenati, D. Schwartzman, M. Gartner, and D. McKell, "Feasibility of a new method for percutaneous occlusion of the left atrial appendage," Circulation, vol. 106:II-619, 2002.

[9] M. A. Zenati and D. Schwartzman, "Impairment of Left Atrial Appendage Mechanical Function Following Electrical Isolation with Epicardial Radiofrequency Bipolar Ablation," J Am Coll Cardiology, vol. 43:432A, 2004

[10]"Olympus Optical Co. Webpage: http://www.olympus.co.jp/indexE.html (Accessed: 9 September 2005)."

[11]K. T. Park and M. Esashi, "An active catheter with integrated circuit for communication and control," presented at Proc. MEMS 99 conf., 1999.

[12]R. Kornbluh, R. Pelrine, J. Eckerle, and J. Joseph, "Electrostrictive polymer artificial muscle actuators," presented at IEEE International Conference on Robotics and Automation, Leuven, Belgium, 1998.

[13]M. I. Frecker and W. M. Aguilera, "Analytical modeling of a segmented unimorph actuator using electrostrictive P(VDF-TrFE) copolymer," Smart Materials and Structures, vol. 13, pp. 82, 2004.

[14]J. Szewczyk, V. de Sars, P. Bidaud, and G. Dumont, "An Active Tubular Polyarticulated Micro-System for flexible Endoscope," in Lecture Notes in Control and Information Sciences, vol. 271 / 2001, Experimental Robotics VII: Springer-Verlag, 2003, pp. 179 - 188.

[15]S. Hirose, "Biologically inspired robots: snake-like locomotors and manipulators," Applied Mechanics Reviews, vol. 48, pp. 27, 1995.

[16]G. Lim, et al., "Multi-link active catheter snake-like motion," Robotica, vol. 14, pp. 499, 1996.

[17]D. Reynaerts, J. Peirs, and H. Van Brussel, "Shape memory microactuation for a gastro-intestinal intervention system," Sensors and Actuators, A: Physical, vol. 77, pp. 157, 1999.

[18]K. Ikuta, M. Nokata, and S. Aritomi, "Biomedical micro robots driven by miniature cybernetic actuator," Proceedings of the IEEE Micro Electro Mechanical Systems, pp. 263, 1994.

[19]Y. Nakamura, A. Matsui, T. Saito, and K. Yoshimoto, "Shapememory-alloy active forceps for laparoscopic surgery," presented at IEEE International Conference on Robotics and Automation, Nagoya, Jpn, 1995.

[20]K. Ikuta, M. Nolata, and S. Aritomi, "Hyper redundant active endoscope for minimally invasive surgery," presented at The first symposium on medical robotics and computer assisted surgery, Pittsburgh, PA., 1994.

[21]K. Ikuta, M. Tsukamoto, and S. Hirose, "Shape memory alloys servo actuator system with electric resistance feedback and application for active endoscope," presented at IEEE International Conference on Robotics and Automation, Philadelphia, PA, USA, 1988.

[22]J. Peirs, D. Reynaerts, H. Van Brussel, G. De Gersem, and H.-W. Tang, "Design of an advanced tool guiding system for robotic surgery," presented at IEEE International Conference on Robotics and Automation, Taipei, Taiwan, 2003.

[23]N. Simaan, R. Taylor, and P. Flint, "A dexterous system for laryngeal surgery," presented at IEEE International Conference on Robotics and Automation, New Orleans, LA, United States, 2004.

[24]J. Vogan, et al., "Manipulation in MRI devices using electrostrictive polymer actuators: With an application to reconfigurable imaging coils," New Orleans, LA, United States, 2004.

[25]P. Dario, et al., "A novel mechatronic tool for computer-assisted arthroscopy," IEEE transactions on information technology in biomedicine, vol. 4, pp. 15-29, 2000.

[26]D. H. Goldman, S. Lum, C. M. Scotti, E. A. Finol, and E. S. Di Martino, "An intensity-based reconstruction protocol for cardiovascular structures," presented at BED 2005 Advances in Bioengineering, Vail, CO., 2005. 